\title{
Taking quantum entanglement out of the lab
}

Paul Gow, James Gates, Rex H. Bannerman, Joshua Nunn, Matthew Posner, et al.

Paul C. Gow, James C. Gates, Rex H. S. Bannerman, Joshua Nunn, Matthew T. Posner, Paolo L. Mennea, Peter G. R. Smith, "Taking quantum entanglement out of the lab," Proc. SPIE 10741, Optics Education and Outreach V, 107410M (14 September 2018); doi: 10.1117/12.2321079

Event: SPIE Optical Engineering + Applications, 2018, San Diego, California, United States 


\title{
Taking quantum entanglement out of the lab
}

\author{
Paul C. Gow ${ }^{* a}$, James C. Gates ${ }^{\mathrm{a}}$, Rex H.S. Bannerman ${ }^{\mathrm{a}}$, Joshua Nunn ${ }^{\mathrm{b}}$, Matthew T. Posner ${ }^{\mathrm{a}}$, \\ Paolo L. Mennea ${ }^{\mathrm{a}}$, and Peter G.R. Smith ${ }^{\mathrm{a}}$ \\ ${ }^{a}$ Optoelectronics Research Centre, University of Southampton, Southampton SO17 1BJ, UK \\ ${ }^{\mathrm{b}}$ Department of Physics, University of Bath, Bath BA2 7AY, UK
}

\begin{abstract}
The Networked Quantum Information Technologies hub (NQIT) is one of the quantum hubs of the UK's flagship scheme, bringing together academia, industry and government. The Optical Engineering and Quantum Photonics group at the University of Southampton's Optoelectronics Research Centre have developed an interactive demonstrator of a key device within NQIT to bring quantum photonics technologies to a wider audience. The system was exhibited at the 2017 UK Quantum Showcase in London to an audience of industry and government. It also featured as part of the Quantum City stand at the 2018 Cheltenham science festival, one of the UK's leading annual science events.
\end{abstract}

Keywords: Photonics outreach, Public engagement with Research, Optical engineering, Integrated quantum photonics

\section{INTRODUCTION}

The Networked Quantum Information Technologies hub (NQIT) is one of the four quantum hubs which make up the UK's flagship scheme, bringing together academia, industry and government to develop the UK as a leader in quantum technology. A key part of this project involves building an optical network to support the construction of a quantum computer.

Due to the complex nature of quantum science it is often seen as a difficult area for engagement and outreach. However, with the increasing interest in quantum technologies and their impact in the future, it is important to engage people with this science and technology. In this paper we present a project by the lead author in developing an interactive Demonstrator of a key optical component of the NQIT project to showcase it to a wider audience. Software was developed to accompany the Demonstrator to show how the real world device works on a single photon level. The system was exhibited at the 2017 UK Quantum Showcase in London to an audience of industry representatives and government policy makers, and was also featured at the 2018 Innovation South showcase at Farnborough conference centre. The demonstrator was part of the Quantum City stand at the 2018 Cheltenham science festival - one of the UK's leading public engagement and science outreach events and was exhibited with a handout for visitors to take away with them explaining the importance of photonics for quantum technologies. This paper discusses the motivation behind the project, the design and construction of the demonstrator, as well as suggestions for a more accessible 'home-made' model for education of quantum entanglement. We will also discuss the reception of the device and any feedback given, as well as lessons learned in this pilot stage of the project.

\section{BACKGROUND}

In this section the context of the quantum technologies programme is presented. The role of the Optical Engineering and Quantum Photonics group (OEQP) in developing a fiber optic Entangler for one of the quantum hubs is discussed. Fabrication challenges and the concept of the device is also presented.

Further author information: (Send correspondence to Paul C. Gow)

E-mail: p.gow@soton.ac.uk, Telephone: +44 2380592959

Optics Education and Outreach V, edited by G. Groot Gregory, Proc. of SPIE Vol. 10741, 107410M

(c) 2018 SPIE · CCC code: 0277-786X/18/\$18 · doi: 10.1117/12.2321079

Proc. of SPIE Vol. 10741 107410M-1 


\subsection{Context}

The $£ 270$ million (GBP) UK national quantum technologies programme ${ }^{1}$ (UKNQTP) is designed to bring together academia, industry and government as part of a strategy to develop the UK as a future leader in quantum technology. ${ }^{2}$ Part of this is an Engineering and Physical Sciences Research Council (EPSRC) funded national network of 4 different Quantum Technology Hubs, with each focusing on a different aspect of quantum science and technology. These include the Sensors and Metrology hub led by Birmingham University, ${ }^{3}$ the Communications Technologies hub led by the University of York, ${ }^{4}$ and the Quantum Enhanced Imaging hub (QuantIC) led by the University of Glasgow. ${ }^{5}$ The fourth hub, and the focus of the work presented here, is the Networked Quantum Information Technologies (NQIT) hub led by the University of Oxford. ${ }^{6}$ This is the largest of the four hubs and is working towards building a hybrid quantum computer linking light and matter technologies for quantum information processing.

Photonics is a major part of modern quantum science, with photons being the main route for mediating quantum interactions, entanglement and measurement. ${ }^{7}$ A recent report by the Photonics Leadership Group revealed that the UK photonics industry is worth around $£ 12.9$ billion (GBP) and employs more than 65,000 people. ${ }^{8} \mathrm{~A}$ report released by the Institute of Physics in May 2018 described the 'health of photonics' and highlighted many of the areas that this technology feeds in to. ${ }^{9}$ Some of these areas include aerospace, communications, health care, food, and energy, with many set to benefit from quantum photonics technologies. Quantum entanglement is frequently mediated through the use of single photons, which immediately means that any loss in components can be catastrophic to quantum systems. Quantum entanglement is also incredibly sensitive, and any external effects can easily destroy these fragile states. Therefore optical engineering is an important aspect for developing the low loss, quantum compatible components and systems that are required for these advanced circuits and communication technologies.

The University of Southampton has played a leading role in photonics and optical technologies research since the 1960's. The Optical Engineering and Quantum Photonics group (OEQP) based within the Optoelectronics Research Centre (ORC) at Southampton have expertise in the design and fabrication of photonic components and devices and collaborate with many of the quantum hubs. The group also has a passion for outreach and public engagement, with its members having published several papers on engagement activities over the past few years. ${ }^{10-12}$ The OEQP group were recognised in 2017 with a 'Highly Commended' awarded for group public engagement by the South East Physics Network (SEPNET). The group are using their expertise in these areas to develop a fiber-optic photonic Entangler system for the NQIT project and engage a wide audience with the science behind quantum technologies and how photonics plays an important role.

\subsection{NQIT Entangler concept and fabrication}

The OEQP group are collaborators within the NQIT hub, developing optical components for quantum applications. One key part of this collaboration is the development and testing of the optical network required for the NQIT hybrid quantum computer. This will consist of solid-state ion traps, based at Oxford, which will be used as computational nodes. Each node needs to be linked with its neighbors via a fiber-optical 'Entangler' capable of causing photons emitted by the trapped ions to undergo quantum entanglement. ${ }^{13}$ This will then allow the ions themselves to become entangled. The system is designed so that it is possible to 'scale-up' by adding further ion traps and optical entangler systems.

The Entangler itself is designed to be constructed with off-the-shelf optical fiber components currently available. This poses a technological challenge in that the majority of components are developed for the current telecommunications market which works with infrared wavelengths of light. The ions used in the NQIT project emit light in the visible blue region, at $422 \mathrm{~nm}$ wavelength, for which many off-the-shelf components show a great deal of loss. With a long history of optical engineering the OEQP groups role is to source and test components for operation at blue wavelengths before assembling these into an optical Entangler system for use with the ion traps. These components are individually tested and assembled inside a 19" rack mount for stability, ease of use and for system expansion through adding further units. The input fibers are attached to the ion traps and guide the photons emitted from the traps to the Entangler unit. Inside, the photons are introduced to each other at a fiber beam splitter. It is here that the photons experience quantum entanglement. If the photons emitted from the ion traps become entangled this filters back to the traps, meaning that the trapped ions become 


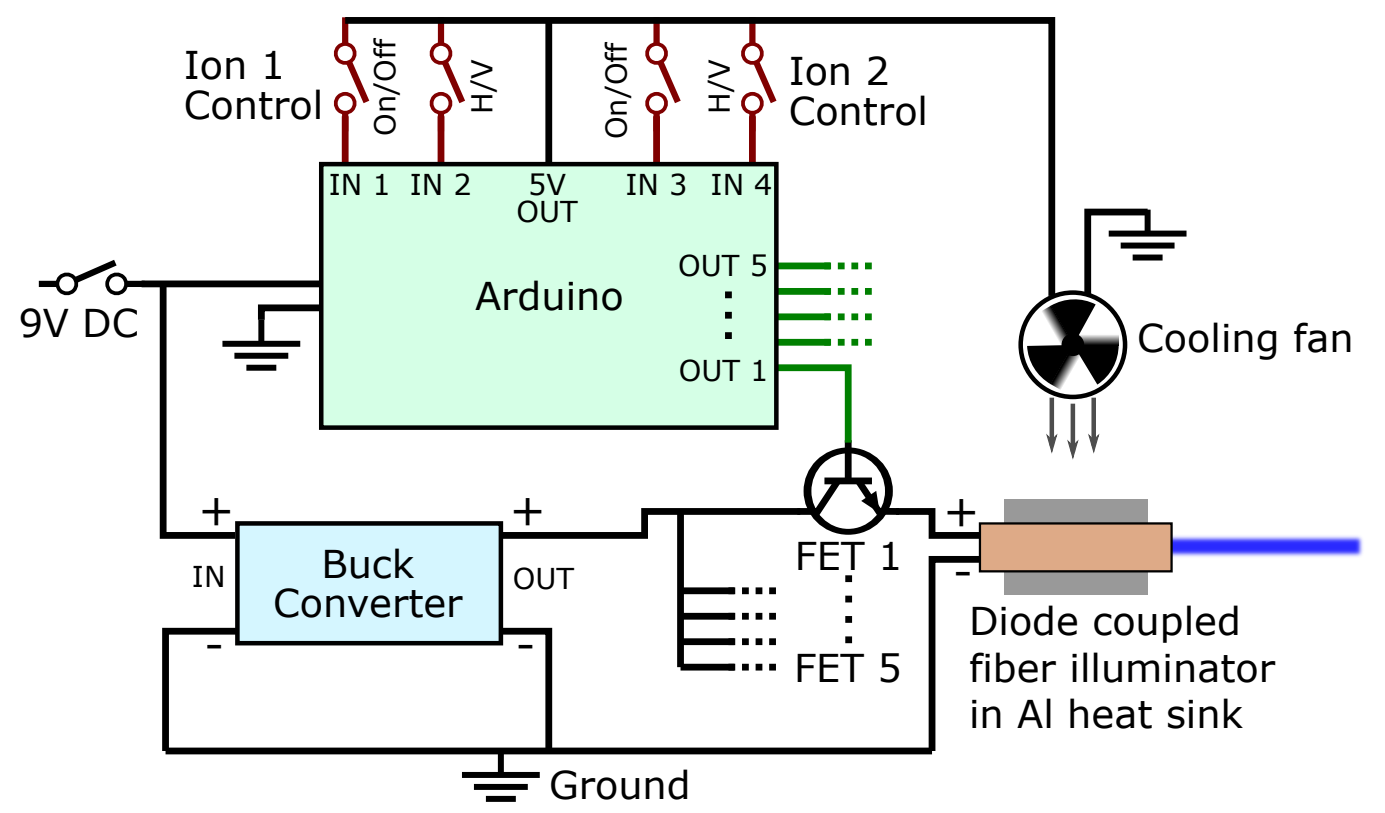

Figure 1. A simplified diagram showing the layout of the circuit for operating the Demonstrator. The system is driven by a $9 \mathrm{~V} \mathrm{DC}, 1 \mathrm{~A}$ power supply which powers the Arduino Uno microcontroller and Buck voltage/current converter. The Arduino is controlled by switches connected to its digital input ports, which are fed from a $5 \mathrm{~V}$ output on the Arduino board itself. The switches turn each channel on and off, and also simulate $\mathrm{H}$ or $\mathrm{V}$ polarisation of light. This also powers the fan used to cool the laser diodes. The Buck converter converts the input supply to provide up to $2.5 \mathrm{~A}$ of current to drive the laser diodes. The output from this is connected in parallel with the five laser diodes through five transistors (FETs). The FETs are gated by the digital outputs from the Arduino.

entangled with each other too. The entangled photons then propagate to a secondary component which splits the photons depending on their polarisation. This stage is used for tuning the ion trap/Entangler system, and for measurement purposes. After the measurement it is possible to tell if entanglement between the ions was successful.

It is clear that the function of this device, and the science behind it, can be quite challenging to explain. It was decided to produce an interactive replica of the real device for engagement purposes. The following sections describe the design and construction of the interactive Demonstrator, as well as accompanying software and a handout designed to highlight the importance of photonics to quantum technology.

\section{HARDWARE AND SOFTWARE IMPLEMENTATION}

The following sections detail the construction of the Demonstrator, how it was assembled and the estimated cost of the device in terms of parts used for manufacturing the device.

\subsection{Hardware}

The hardware was designed to replicate the real-world Entangler unit that would be used in the fundamental physical experiments of the NQIT project. Blue laser diode illuminated optical fibers were used, along with an electronic circuit to drive them and Arduino for control. The electronics were housed within a 19" rack mountable unit with the illuminated fibers feeding through to an additional unit above. The construction of the unit, and its operation, are discussed in the following sections.

\subsubsection{Construction}

The equipment required for constructing the unit is listed as follows, with total costs displayed in GB Pounds. The total equipment cost was approximately £685.43. The cost of staff time taken to assemble the device is not taken into account here. 
- 2 x 1U 19" rack mountable units (RS components PF-19), used for housing the optics and electronics and for operation of the demonstrations. (£95.74)

- $5 \mathrm{x}$ Toggle switches for activating the device and controlling circuit. (£5.28)

- 5 x Blue laser diode illuminated fibers (World Star Tech ML5-20G-450-2M), which relate directly to the optical fiber component of the real Entangler. These illuminate to show the paths that photons would take in the device. $(£ 450)$

- $1 \mathrm{x}$ Arduino Uno, microcontroller for control of the electronics to allow the fibers to light up under certain combinations of switches. (£20.70)

- 1 x Buck Voltage/Current converter, for electronically driving the illuminated fibers and LED's on the unit. (£1.20)

- $5 \mathrm{x}$ Transistors (FET’s), used for gating the illuminated fibers with the Arduino. (£4.50)

- $1 \mathrm{x}$ Piece of clear Polycarbonate, for the lid of the unit so that the fibers can be viewed, but are also protected. (£38.16)

- 1 x USB port and cable, for reprogramming Arduino without having to dismantle the box. (£8.11)

- $2 \mathrm{x}$ Die cast boxes to represent the beamsplitter components. (£11.74)

- Other components such as; resistors, cables, electronic connectors, cable glands, electronic prototype board, LED's, foam etc. (approx. £50)

The electronic components were assembled as shown by the circuit diagram in figure (1). The entire circuit was driven through a DC adapter which converted the $240 \mathrm{~V}$ AC mains supply to a $9 \mathrm{~V} \mathrm{DC}$ supply of less than $1.2 \mathrm{~A}$, through a switch to control power to the entire device. The five illuminated fibers are driven by blue
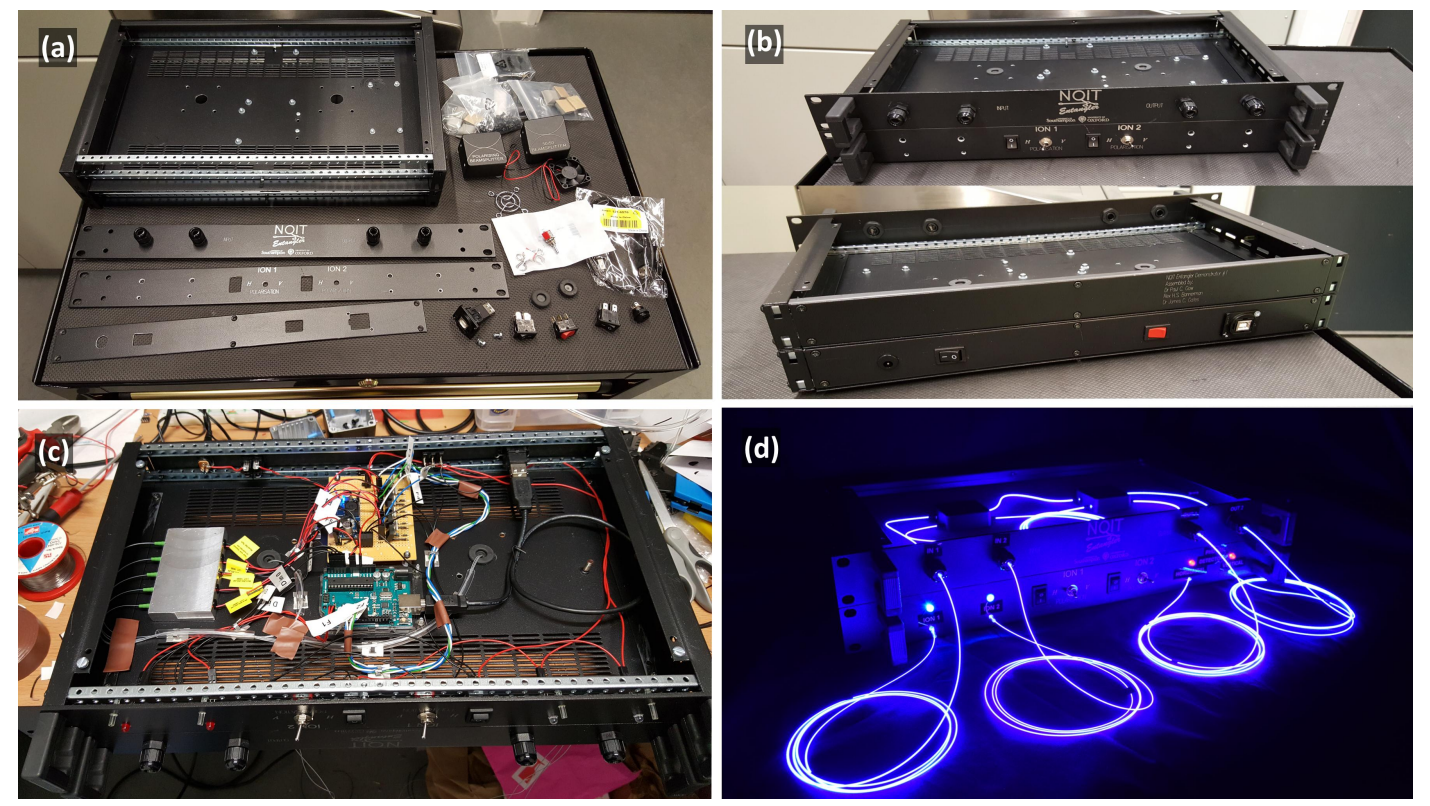

Figure 2. Collection of images showing the construction of the Demonstrator. Fig (a) shows the parts layed out prior to assembly. Fig (b) show the front (top) and back (bottom) of the assembled unit prior to electronics installation. Fig (c) shows the installation of the electronics in the lower rack unit, with diode coupled illuminated fibers on the left, Arduino at the centre bottom, and the FETs and Buck converter centre top. Video (d) shows the completed Demonstrator unit in operation. http://dx.doi.org/10.1117/12.2321079.1 

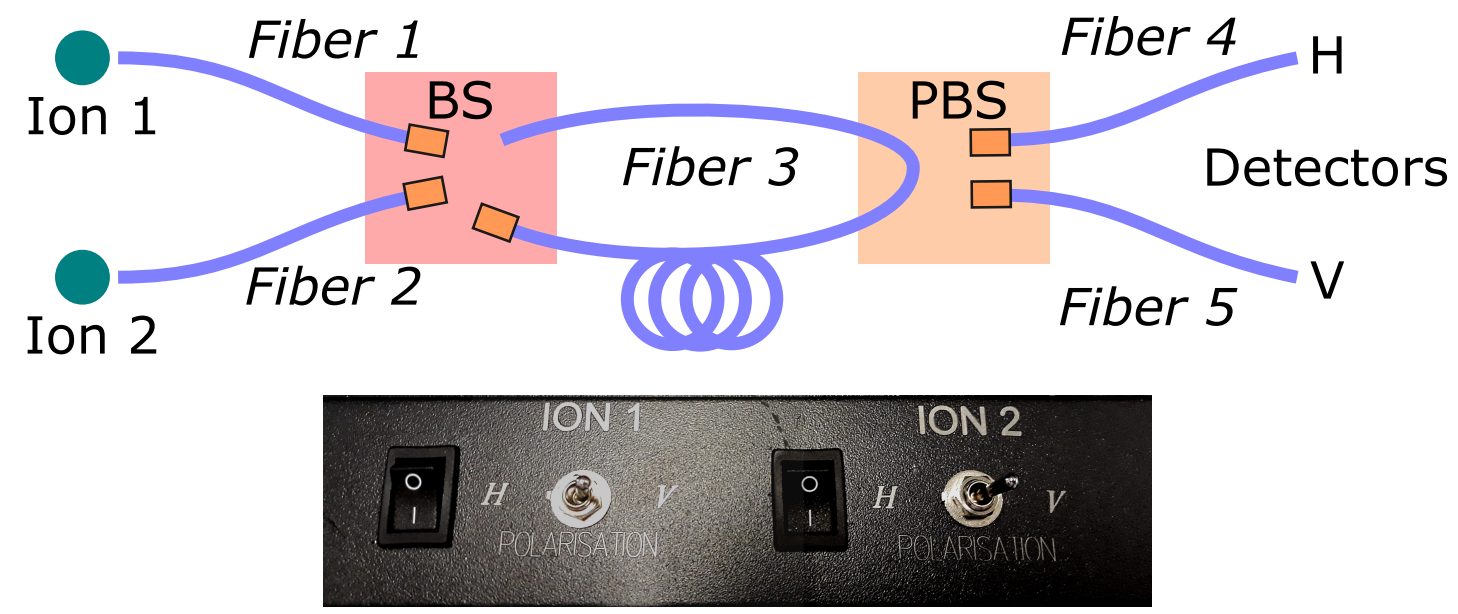

Figure 3. Schematic of the Demonstrator operation. This shows how the illuminated fibers are routed out of the beamsplitter (BS) and polarising beamsplitter (PBS), and the way in which they will light up depending on the switches. The lower image is of the switches on the front panel.

laser diodes which are directly coupled and provided as a complete unit from World Star Tech. These were connected electrically in parallel through separate FETs to a buck voltage/current converter, which scaled the input current to that accepted by the fibers. The Arduino was powered directly from the input $9 \mathrm{~V}$ supply and was programmed to accept four input signals (four switches) and respond accordingly. Five outputs from the Arduino were used to gate the FETs allowing the fibers to illuminate with respect to the input signals. The Arduino outputs were also programmed to give the fibers a pulsing effect when illuminated.

The two rack mount units were assembled and attached together. The electrical components were installed in the lower of the two units, shown in figure (2c). An electrical port and a switch was installed on the rear of the unit for connection to the $9 \mathrm{~V}$ DC power supply. A USB port was also installed and connected internally to the Arduino to allow reprogramming without requiring disassembly of the unit. The four switches for control of the device were installed on the front panel of the lower unit. LEDs were also installed on this front panel to indicate when the circuit was activated, and were wired into the switches. The front and back panels are shown in figure $(2 b)$.

The parts of the illuminated fibers which housed the laser diodes were also installed in the lower unit. A block of aluminium was machined to hold these five components and to act as a heat sink. A small fan was fixed inside the casing next to this block to ensure constant airflow and cooling of the diodes when under operation. The illuminated fibers were then fed through holes into the unit above.

The front panel of the top unit was machined to hold four cable glands, which the illuminated fibers can pass out of for demonstrations. The top unit was also filled with a soft black foam to protect the fibers which lay on top of it. The holes which the fibers pass through from the lower unit were covered with small die-cast boxes which were patterned and labelled as 'beamsplitter' and 'polarising beamsplitter' to indicate the two fiber components used in the real Entangler device. The fibers then fed out of small holes drilled in the sides of these boxes. The top unit was capped with a piece of Perspex cut to size, to protect the fibers within and allow viewing. Both front panels of the top and bottom unit were engraved with a $\mathrm{CO}_{2}$ laser, labeling the function of the switches, inputs and outputs, and including the NQIT logo. Figures (2a to c) show the assembly of the unit and figure $(2 \mathrm{~d})$ shows the completed device in operation.

\subsubsection{Operation}

The Demonstrator was designed to mimic the real-world Entangler unit that accepts photons from two separate ion traps. Figure (3) shows a schematic of the device operation. The switches to control the channels 'Ion 1' and 'Ion 2' are designed to mimic inputs from two Ion traps which are individually controlled. These switches primarily control the illumination of fibers 1, 2 and 3. They also control the illumination of fibers 4 and 5 , 


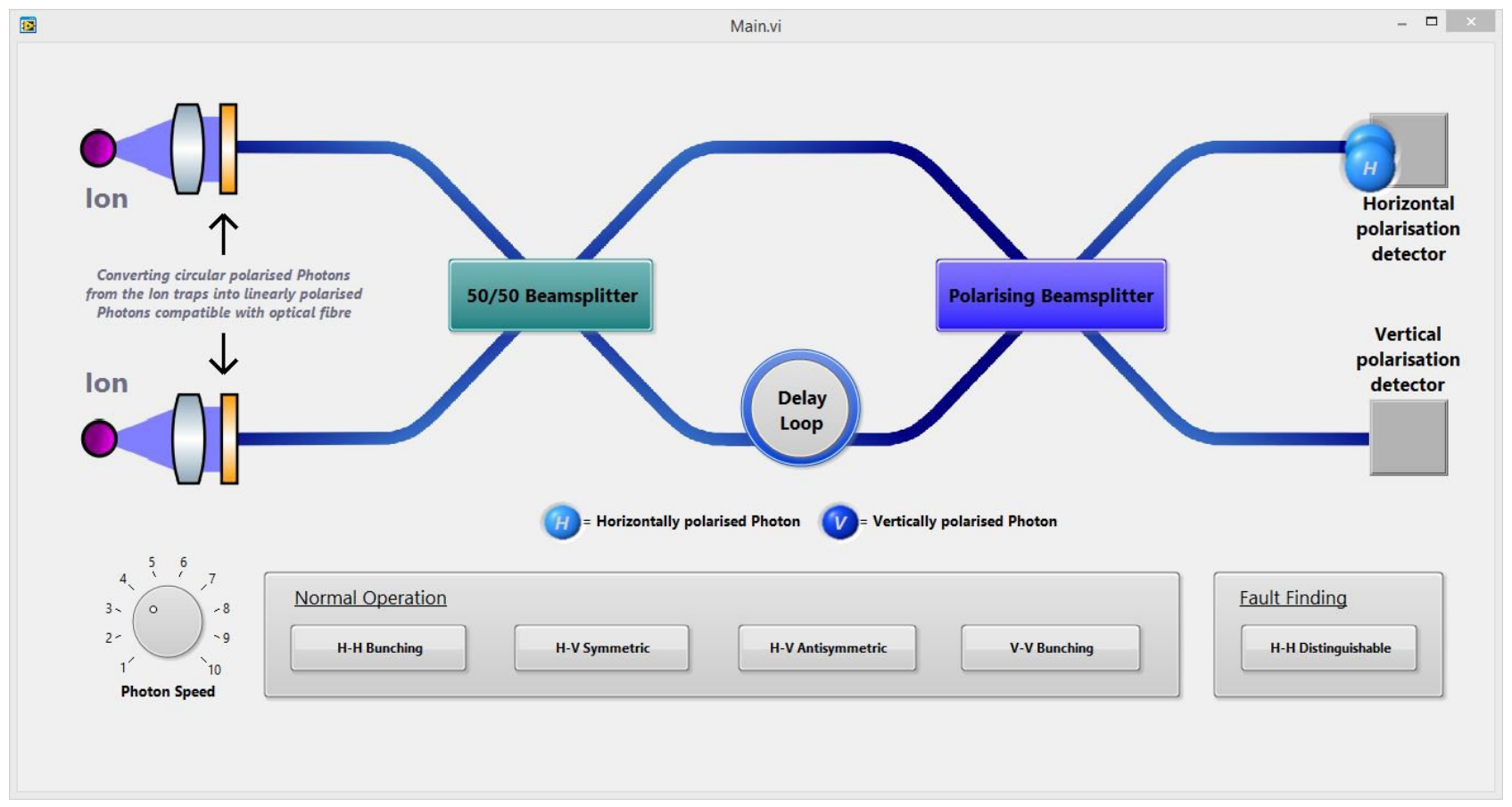

Figure 4. Video of the LabVIEW developed software to support the Demonstrator. The design mimics the experimental setup, with two ion traps emitting photons which enter the fiber optic circuit. The buttons at the bottom of the image select different entanglement scenarios which are then played out by the blue circular 'photons' over the diagram. http://dx.doi.org/10.1117/12.2321079.2

however which of these fibers illuminates is controlled by the other switches with positions labeled ' $\mathrm{H}$ ' and ' $\mathrm{V}$ ' to denote the polarisation of the photons as either horizontal or vertical.

The best way of understanding how the Demonstrator works is by considering one channel at a time. For instance with the 'Ion 1' switch activated fiber 1 will be illuminated. This passes into the first black box which is presented as a beamsplitter. A beamsplitter would take the light from an input and split the light 50/50 into two outputs. As both outputs of the beamsplitter would be illuminated whether 'Ion 1' or 'Ion 2' is activated, we use only fiber 3 in a loop between the two black boxes to give the impression of two output fibers. The second black box is presented as a polarising beamsplitter. This directs the light into an output depending on what polarisation it is, horizontal or vertical. The polarisation of the light is controlled by the ' $\mathrm{H}$ ' and ' $\mathrm{V}$ ' switch. For instance, if the switch is set to ' $\mathrm{H}$ ' then fiber 4 will illuminate. If the switch is set to ' $\mathrm{V}$ ' then fiber 5 illuminates.

This is similar to the outcome when the 'Ion 2' switch is activated, with fiber 2 instead of fiber 1 illuminated. With both 'Ions' activated fibers $1,2 \& 3$ will illuminate and fibers $4 \& 5$ are controlled by the two ' $\mathrm{H}$ ' and ' $\mathrm{V}$ ' switches. The Arduino handles all of these switch inputs and activates the fibers accordingly.

\subsection{Supporting software}

The Demonstrator works well to show the function of the device in terms of how different inputs affect the outputs, however the real Entangler operates at the single photon level. To describe the function of the Demonstrator in terms of single photons some supporting software was developed using National Instruments LabVIEW. The resulting GUI is shown in figure (4).

The software was designed to mirror the Demonstrator design, with different cases for $\mathrm{H}$ and $\mathrm{V}$ entanglement for the different Ions, and a graphic representation of the fibers and beamsplitters. When one of the buttons at the bottom is pressed, two 'photons' labelled ' $\mathrm{H}$ ' or ' $\mathrm{V}$ ' travel from the 'ion traps' at the far left of the image towards the first 'beamsplitter'. Here they interact depending on the button selected before propagating to the 'polarising beamsplitter' to interact again. They then move onwards to the 'detectors' which flash depending on 
how many 'photons' are detected. Whilst supporting the Demonstrator, the software is targeted at explaining the function of the Entangler to a technical audience. The means that it may not be suitable for all engagement audiences and there are some scenarios when the Demonstrator alone would be most suitable.

\section{ENGAGEMENT WITH THE DEMONSTRATOR}

The Demonstrator was first exhibited along with the accompanying software at the Quantum Technologies Showcase on the $22^{\text {nd }}$ of November 2017, held at the Queen Elizabeth II conference centre in Westminster, London UK. The annual showcase is designed to demonstrate the technological progress arising from the UK National Quantum Technologies Programme, its relevance to commercial sectors and the collaborative nature of the programme involving academia, industry and government partners. As such, the audience at this event mainly consisted of academics, company representatives, and policy makers. The Demonstrator was exhibited as part of the OEQP groups stand, and featured alongside other examples of engineering and photonic components developed by the group (figure 5a). For this event an estimated 70 people were engaged with on the stand.

The second event to exhibit the Demonstrator was the Innovation South showcase event hosted by Innovate UK on the $27^{\text {th }}$ March 2018 at the Farnborough conference centre. There were approximately 20 stands from companies, universities, and funding bodies there. Around 400 people registered for the day, with an estimated 40 people engaged on the entangler demonstrator alone during the course of the day. In this instance the hardware unit was displayed alone without the accompanying software. The demonstrator mainly sparked conversation on what a photon was and the strange aspects of quantum science. Most comments were focussed around what quantum would be useful for: building blocks for quantum computers to enable faster parallel processing, drug design, and security.

The Demonstrator also featured as part of the Quantum City stand at the Cheltenham science festival, which ran from the $5^{\text {th }}$ to the $10^{\text {th }}$ of June 2018. A recent public dialogue commissioned by EPSRC indicated that the public would better relate to quantum research if it was presented through familiar applications and situations. ${ }^{14}$ The idea of a 'Quantum City' stand was developed for this purpose and will continue to exhibit at many science related festivals and events. At the Cheltenham Science Festival the stand exhibited different demonstrations from the various partners of the UKNTQP on each day of the festival between 10 am and $5 \mathrm{pm}$, with the $10^{\text {th }}$ of June 2018 focussing on the NQIT hub. The Demonstrator was exhibited alongside other demonstrations of ion traps and interactive games design to educate about quantum science and what makes quantum computers unique (figure 5b). The number of people engaged with at the stand was measured with a finger-clicker over the course of an hour between 11:30 am and 12:30 pm. During this time 62 people were engaged with at the stand, therefore giving an estimated 430 interactions during the day. The handout shown in figure (6a) was
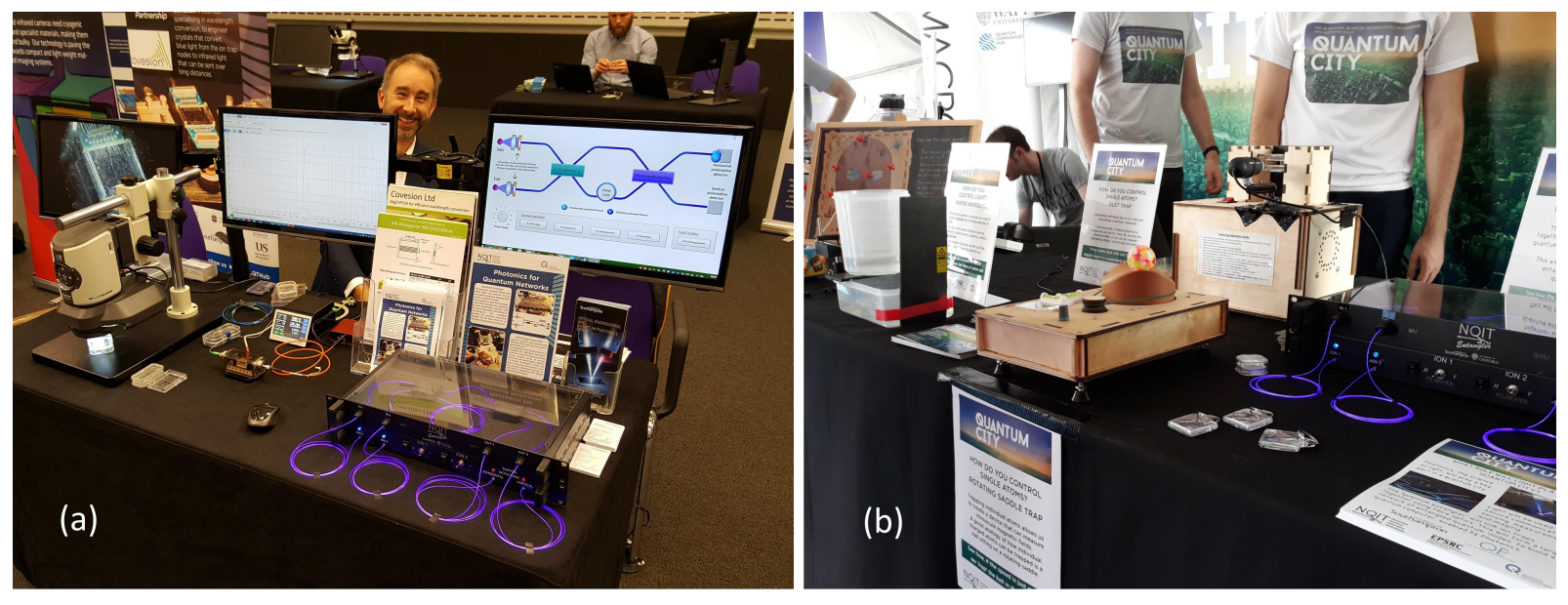

Figure 5. Images of the Demonstrator on exhibition at the National Quantum Technologies Showcase (a), and on the Quantum City stand at the Cheltenham Science Festival (b). 
(a)

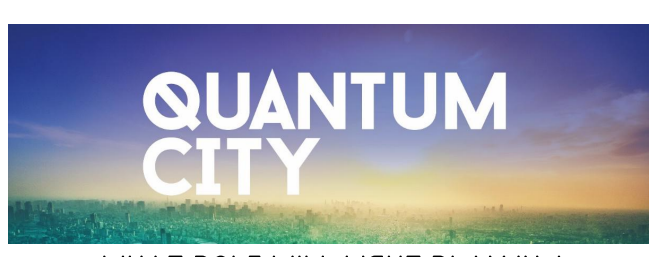

WHAT ROLE WILL LIGHT PLAY IN A

QUANTUM CITY?

Photonics, the science of light, will play a key part in a quantum city.
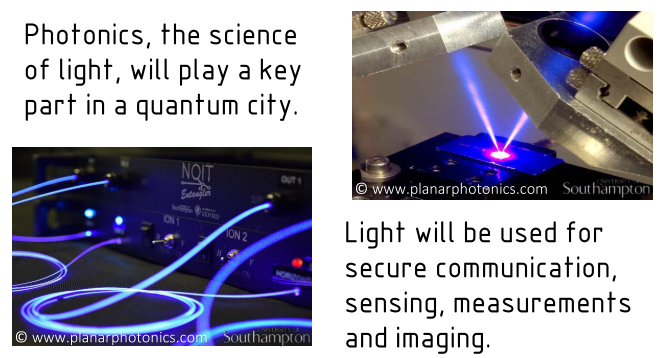

Light will be used for secure communication, sensing, measurements and imaging.

The 'quantum entangler' will link ion traps together using fibre optics. This will form a large network of entangled quantum 'nodes' to build a quantum computer, enabled by Photonics.

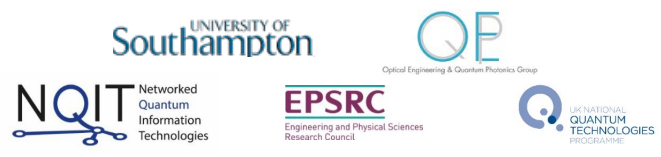

(b)

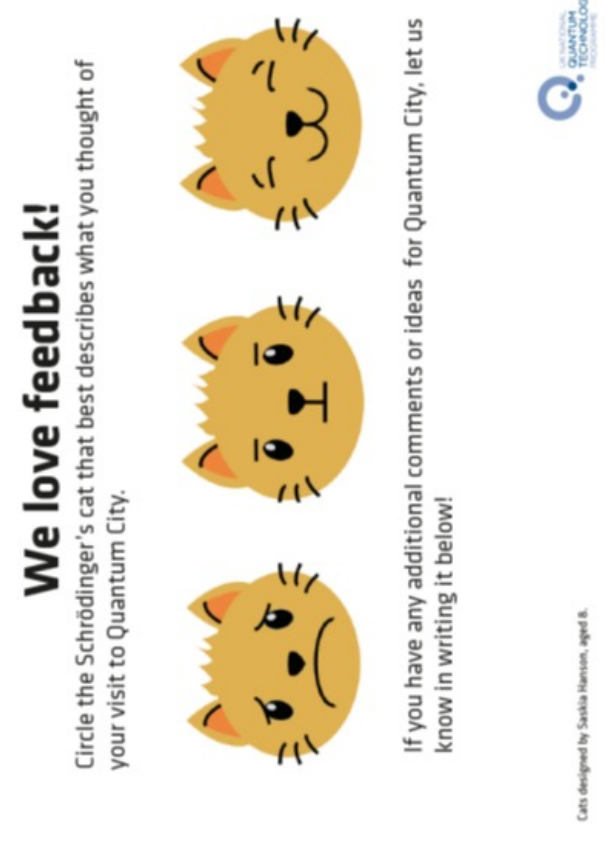

Figure 6. Examples of the handouts used for the Quantum City stand. Fig (a) shows the flyer developed to show how photonics and the entangler are important for the NQIT project. Fig (b) shows an example of the feedback form used for the Quantum City stand. People engaged with at the stand are encouraged to provide feedback by circling either the happy, neutral, or sad cat to indicate their experience. There was space below for any additional comments to be filled in.

produced as something for people to take away with them that explained the importance of photonics, light and the Entangler to a quantum city of the future. People interacting with the stand were encouraged to fill out a "Schrodingers Cat" feedback postcard with space for optional comments (figure $6 \mathrm{~b}$ ), and $95 \%$ of responses highlighted the happy cat symbol.

\section{PERSPECTIVES AND OUTLOOK}

This section highlights some of the lessons learned during the construction and exhibition of the Demonstrator, and looks ahead to further opportunities for engagement in the near future. A way of achieving an alternative demonstration for education of entanglement at a fraction of the cost is also discussed.

\subsection{Lessons learned}

The initial concept of the Demonstrator was to construct a replica of the Entangler that would be used in experiments in the NQIT hub. This replica was to be used to explain the function of the real world device and help to explain the importance of photonics to quantum research and the NQIT hub. Upon construction it was realised that, while the Demonstrator showed the general concept of the device, it did not do well explaining the science on a single photon level in which the real world Entangler would be operating. For this reason some software was developed to be exhibited alongside the Demonstrator which would show the different means of operation for single photons. However, the combination of the Demonstrator and software make this quite a 'high level' engagement tool. This worked well for the UK Quantum Technologies Showcase and the Innovation South Showcase, which were targeted at company representatives, academics and government officials, however this did not work well as an engagement tool for the general public. Therefore a handout was developed for 


\section{Step 1: Photon emission}

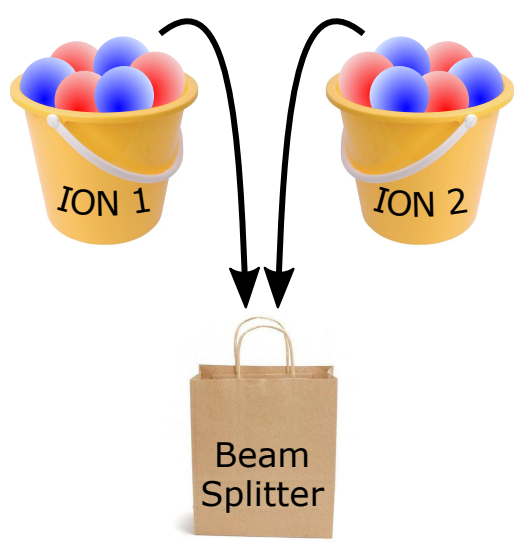

Step 2: Measurement

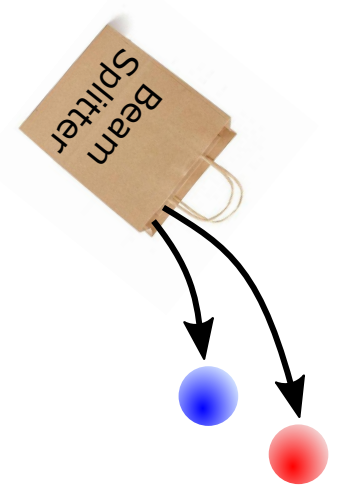

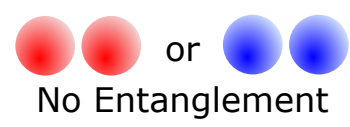

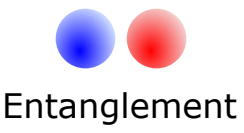

Figure 7. Example of a simple scheme for demonstrating entanglement. In step one a 'photon' from each 'Ion' is selected blindly and at random, and placed into the 'beamsplitter'. In step 2 the 'photons' are measured. If the 'photons' match we know the state of the 'Ions' and do not achieve entanglement. If they do not match we can't tell from which 'Ion' each 'photon' came and entanglement is achieved.

the Quantum City stand to inform about the use of the entangler and the importance of photonics for quantum technology. The Demonstrator was also intended to be interactive, with people encouraged to try out the switches and use the software to explore the device operation. However, it often resulted in the person exhibiting the device operating both switches and software whilst explaining the function. Due to the delicate nature of the illuminated fibers this was also exhibited as a 'hands-off' device to the public, which was not the original intention. The construction of the device, whilst not costing a large amount, could prove costly to replicate on a minimal budget. The Demonstrator was also time consuming to construct and therefore could be inaccessible for many people to replicate. The following section discusses an alternative option for demonstrating quantum entanglement in a 'hands-on' way that would prove cheap and quick to implement.

\subsection{Hands-on entanglement}

A concept for an interactive, hands-on method for describing photon entanglement which could be exhibited on its own or alongside the Demonstrator is introduced here and shown in figure (7). The general principle behind the real world Entangler is that photons emitted by two different ions are brought together at a beamsplitter to achieve entanglement. An important aspect of entanglement is that you must not know which photon came from which ion, otherwise you will know which state each ion is in thus breaking the quantum entanglement. The simplest way to demonstrate this concept would be through the use of coloured or numbered tokens being selected from two separate containers, or even dispensed by two 'bingo ball' machines. For instance, assuming the use of red and blue balls to signify the different photon states, two buckets to hold a mixture of the balls representing the ions, and an opaque bag to represent the beamsplitter, the following could be a demonstration of entanglement:

- First a 'photon' ball is selected blindly from one 'Ion' bucket and placed into the 'beamsplitter' bag.

- Next a 'photon' ball is selected blindly from the other 'Ion' bucket and placed into the 'beamsplitter' bag.

- The 'beamsplitter' bag is opened and the 'photons' are compared.

If the balls are both red (blue) we know that both buckets produced a red (blue) ball, therefore we know which state each ion is in and so they are not entangled. If one ball is red and the other is blue then we know that each bucket must have produced a red or blue ball, but we don't know which. In this case we can say that the buckets are in a state of entanglement. This method is hands-on, simple to explain, and is easy to implement for a low cost. 
The Demonstrator and software will feature again at the UK Quantum Technologies Showcase 2018, and will play a part in any engagement the Optical Engineering \& Quantum Photonics group undertakes. The Quantum City stand will appear at several more events over the next year, with the intention that the Demonstrator will be featured at some of the events. A simple hands-on demonstration like that discussed above will accompany the Demonstrator at these events.

\section{CONCLUSION}

A replica of the Entangler to be used in experiments in the NQIT hub was constructed to explain the function of the real world device and help to explain the importance of photonics to quantum research and the NQIT hub. Software was developed to be exhibited alongside the device to show the different means of operation for single photons. The Demonstrator was exhibited to members from academia, industry and government at the UK Quantum Technologies Showcase 2017 and the Innovation South Showcase 2018. It was also exhibited to the general public at the Cheltenham Science Festival 2018 as part of the Quantum City stand, an initiative by the UKNQT hubs to engage people in quantum science and find out what a 'quantum city' of the future might look like. The total number of people engaged with the Demonstrator so far number approximately 540 . The Demonstrator proved to be successful in highlighting the importance of photonics and fiber technologies in a quantum city and the growing quantum sector as a whole. The device proved to be 'hands-off' during demonstrations and could prove inaccessible for many people to replicate, therefore a supplementary option for demonstrating quantum entanglement in a 'hands-on' and cost effective way was discussed, with plans to implement this alongside the demonstrator at future events.

\section{ACKNOWLEDGEMENTS}

The authors would like to acknowledge EPSRC grant EP/M013243/1 for funding this activity.

\section{REFERENCES}

[1] "UK national quantum technologies programme website." Available at http://uknqt.epsrc.ac.uk/ (Accessed 14/07/2018).

[2] The quantum technologies strategic advisory board, "National strategy for quantum technologies." EPSRC \& Innovate UK, 23 March 2015, Available at https://epsrc.ukri.org/newsevents/pubs/ quantumtechstrategy/. (Accessed: 12 July 2018).

[3] "UK National Quantum Technology Hub Sensors and Metrology." Available at https://www . quantumsensors.org/ (Accessed 19/07/2018).

[4] "Quantum communications hub." Available at https://www.quantumcommshub.net/ (Accessed $19 / 07 / 2018)$.

[5] "Quantum enhanced imaging hub." Available at https://quantic.ac.uk/ (Accessed 19/07/2018).

[6] "Networked quantum information technologies." Available at http://nqit.ox.ac.uk/ (Accessed $19 / 07 / 2018)$.

[7] O’brien, J. L., Furusawa, A., and Vučković, J., "Photonic quantum technologies," Nature Photonics 3(12), 687 (2009).

[8] Lincoln, J. and Wasley, M., "UK Photonics: The Hidden Economic Engine." Photonics UK, 16 May 2018, Available at https://photonicsuk.org/wp-content/uploads/2018/05/UK_Photonics_The_ Hidden_Economy.pdf. (Accessed: 12 June 2018).

[9] The Institute of Physics, "The health of photonics." IOP, May 2018, Available at https://www.iop.org/ publications/iop/2018/file_71498.pdf. (Accessed: 12 June 2018).

[10] Posner, M. T., John, P. V., Standen, D., Wheeler, N. V., Van Putten, L. D., Soper, N., Parsonage, T. L., Wong, N. H., and Brambilla, G., "Reflecting photonics: reaching new audiences through new partnershipsIYL 2015 and the Royal Horticultural Society Flower Show," in [Optics Education and Outreach IV], 9946, 994603, International Society for Optics and Photonics (2016). 
[11] Posner, M. T., John, P. V., Wong, N. H., Mittal, V., and Nunez-Velazquez, M. M., "From school classes to UNESCO: IYL-enabled environments for tackling the STEM skills shortage through student-led outreach," in [Optics Education and Outreach IV], 9946, 994607, International Society for Optics and Photonics (2016).

[12] Posner, M. T., Jantzen, A., van Putten, L. D., Ravagli, A., Donko, A. L., Soper, N., Wong, N. H., and John, P. V., "Cathedral outreach: student-led workshops for school curriculum enhancement in non-traditional environments," in [Education and Training in Optics and Photonics], 1045207, Optical Society of America (2017).

[13] Olmschenk, S., Matsukevich, D., Maunz, P., Hayes, D., Duan, L.-M., and Monroe, C., "Quantum teleportation between distant matter qubits," Science 323(5913), 486-489 (2009).

[14] Busby, A., Digby, A., and Fu, E., "Quantum technologies public dialogue report." Kantar Public, 13 June 2018, Available at https://epsrc.ukri.org/newsevents/pubs/ epsrc-quantum-technologies-public-dialogue-full-report/. (Accessed: 12 July 2018). 